\title{
Overcoming trastuzumab resistance with nordihydroguaiaretic acid?
}

\author{
Kadri Altundag ${ }^{1}$, Ozden Altundag ${ }^{2}$, Ebru Karakaya ${ }^{3}$, and Serap Akyurek ${ }^{4}$ \\ ${ }^{1}$ Department of Medical Oncology, Hacettepe University Medical School, Ankara, Turkey; ${ }^{2}$ Ozden Altundag, 8181 \\ Fannin Street No. 728, Houston, TX, 77054, USA; ${ }^{3}$ Department of Radiation Oncology, Hacettepe University Medical \\ School, Ankara, Turkey; ${ }^{4}$ Department of Radiation Oncology, Ankara University School of Medicine, Ankara, Turkey
}

Key words: breast cancer, insulin growth factor receptor, nordihydroguaiaretic acid, resistance, trastuzumab

We congratulate Youngren and his colleagues for their fascinating study showing that nordihydroguaiaretic acid (NDGA) inhibits the IGF-1 and c-erbB2/HER2/ neu receptors and suppresses growth in breast cancer cells [1]. Trastuzumab (Herceptin) is a recombinant humanized monoclonal antibody, which targets an epitope in the extracellular domain of the HER2 protein. Preclinical models demonstrated that this antibody has significant anti-tumor activity as a single agent and has synergy with certain chemotherapeutic drugs. Phase II and III clinical trials performed in women with metastatic breast cancer that overexpress HER2 have shown that trastuzumab has clinical activity when used as first-, second- or third-line monotherapy, and improves survival when used as first-line therapy in combination with chemotherapy [2]. Unfortunately $30-40 \%$ of HER2overexpressing cancer cells respond to treatment with trastuzumab, and the clinical benefit of the drug is limited by the fact that most cancers become resistant to trastuzumab therapy in less than 12 months [3]. Mechanism of resistance to trastuzumab therapy includes overexpression of the insulin-like growth factor receptor (IGF-1R), downregulation of p27kip1 and loss of PTEN [4]. Moreover, $\mathrm{Lu}$ et al. [3,5] showed that in breast cancer cell models that overexpress HER-2, an increased level of IGF-IR signaling seems to interfere with the action of trastuzumab by reducing p27 (Kip1) protein level by increased degradation. Therefore, use of NDGA alone or combination with trastuzumab may prevent or delay development of resistance to trastuzumab in breast cancer cells. Further in vitro and in vivo studies are warranted to support this proposal.

\section{References}

1. Youngren JF, Gable K, Penaranda C, Maddux BA, Zavodovskaya M, Lobo M, Campbell M, Kerner J, Goldfine ID: Nordihydroguaiaretic acid (NDGA) inhibits the IGF-1 and c-erbB2/ HER2/neu receptors and suppresses growth in breast cancer cells. Breast Cancer Res Treat 2005 Aug 23 [Epub ahead of print]

2. Yeon $\mathrm{CH}$, Pegram MD: Anti-erbB-2 antibody trastuzumab in the treatment of HER2-amplified breast cancer. Invest New Drugs 23: 391-409, 2005

3. Lu Y, Zi X, Zhao Y, Mascarenhas D, Pollak M: Insulin-like growth factor-I receptor signaling and resistance to trastuzumab (Herceptin). J Natl Cancer Inst 93: 1852-1857, 2001

4. Altundag K, Esteva FJ, Arun B: Monoclonal antibody-based targeted therapy in breast cancer. Curr Med Chem Anti-Cancer Agents 5: 99-106, 2005

5. Lu Y, Zi X, Pollak M: Molecular mechanisms underlying IGFI-induced attenuation of the growth-inhibitory activity of trastuzumab (Herceptin) on SKBR3 breast cancer cells. Int J Cancer 108: 334-341, 2004

Address for offprints and correspondence: Ozden Altundag, Ozden Altundag, 8181 Fannin Street No. 728, Houston, TX, 77054, USA; Tel.: +1-713-7950438; Fax: + 1-713-794-4385; E-mail: altundag@sbcglobal. net 